\title{
Treatment of post-meniscectomy knee symptoms with medial meniscus replacement results in greater pain reduction and functional improvement than non-surgical care
}

\author{
Kenneth R. Zaslav ${ }^{1}$ - Jack Farr ${ }^{2} \cdot$ Richard Alfred $^{3} \cdot$ R. Maxwell Alley $^{3} \cdot$ Michael Dyle $^{4} \cdot$ Andreas H. Gomoll $^{5}$. \\ Christian Lattermann ${ }^{6}$. Brian P. McKeon ${ }^{7}$. Christopher C. Kaeding ${ }^{8} \cdot$ Thomas Giel $^{9}$. Elliott B. Hershman ${ }^{10}$
}

Received: 28 August 2020 / Accepted: 6 April 2021 / Published online: 21 April 2021

(c) The Author(s) 2021

\begin{abstract}
Purpose Partial meniscectomy is a common orthopedic procedure intended to improve knee pain and function in patients with irreparable meniscal tears. However, 6-25\% of partial meniscectomy patients experience persistent knee pain after surgery. In this randomized controlled trial (RCT) involving subjects with knee pain following partial meniscectomy, it was hypothesized that treatment with a synthetic medial meniscus replacement (MMR) implant provides significantly greater improvements in knee pain and function compared to non-surgical care alone.

Methods In this prospective, multicenter RCT, subjects with persistent knee pain following one or more previous partial meniscectomies were randomized to receive either MMR or non-surgical care. This analysis evaluated the 1-year outcomes of this 2-year clinical trial. Patient-reported knee pain, function, and quality of life were measured using nine separate patient-reported outcomes. The primary outcomes were the pain subscale of the Knee injury and Osteoarthritis Outcome Score (KOOS) and the average of all five KOOS subscales (KOOS Overall). Treatment cessation was defined as permanent device removal in the MMR group and any surgical procedure to the index knee in the non-surgical care group.

Results Treated subjects had a median age of 52 years old (range 30-69 years) and one or more previous partial meniscectomies at a median of 34 months (range 5-430 months) before trial entry. Among 127 subjects treated with either MMR $(n=61)$ or non-surgical care $(n=66), 11$ withdrew from the trial or were lost to follow-up (MMR, $n=0$; non-surgical care, $n=11)$. The magnitude of improvement from baseline to 1 year was significantly greater in subjects who received MMR in both primary outcomes of KOOS Pain $(P=0.013)$ and KOOS Overall $(P=0.027)$. Treatment cessation was reported in $14.5 \%$ of non-surgical care subjects and only $4.9 \%$ of MMR subjects (n.s.).
\end{abstract}

Conclusion Treatment with the synthetic MMR implant resulted in significantly greater improvements in knee pain, function, and quality of life at 1 year of follow-up compared to treatment with non-surgical care alone.

Level of evidence I.

Keywords Partial meniscectomy · Medial meniscus · Meniscus lesion · Meniscus tear · Implant $\cdot$ Replacement · NUsurface $\cdot$ Non-surgical care $\cdot$ RCT $\cdot$ KOOS

Kenneth R. Zaslav

Kzaslav@gmail.com

1 Ortho Virginia, Virginia Commonwealth University, 7858 Shrader Road, Richmond, VA 23294, USA

2 OrthoIndy, Greenwood, IN, USA

3 The Bone and Joint Center, Albany, NY, USA

4 Telos Partners, LLC, Denver, CO, USA

5 Hospital for Special Surgery, New York, NY, USA
6 Brigham and Women's Faulkner Hospital Orthopaedic Center, Boston, MA, USA

7 Boston Sports and Shoulder Center, Waltham, MA, USA

8 The Ohio State University Wexner Medical Center Sports Medicine, Columbus, OH, USA

9 OrthoSouth, Memphis, TN, USA

10 Northwell Health Physician Partners, Orthopaedic Institute at Lenox Hill, New York, NY, USA 


$\begin{array}{ll}\text { Abbreviations } \\ \text { MMR } & \begin{array}{l}\text { Medial meniscus replacement } \\ \text { VENUS }\end{array} \\ & \begin{array}{l}\text { Verifying the Effectiveness of the } \\ \text { NUsurface }\end{array} \\ \text { RCT } & \text { System } \\ \text { BMI } & \text { Randomized controlled trial } \\ \text { MRI } & \text { Body mass index } \\ \text { KOOS } & \text { Magnetic resonance imaging } \\ & \text { Knee Injury and Osteoarthritis Outcome } \\ \text { ADL } & \text { Score } \\ \text { QOL } & \text { Activities of daily living } \\ \text { VAS } & \text { Visuality of life } \\ \text { WOMET } & \text { Western Ontario Meniscal Evaluation Tool } \\ \text { IKDC } & \text { International Knee Documentation } \\ & \text { Committee } \\ \text { PCU } & \text { Polycarbonate-urethane } \\ \text { UHMWPE } & \text { Ultrahigh molecular weight polyethylene } \\ \text { NS } & \text { Non-surgical } \\ \text { MD } & \text { Mean difference } \\ \text { TE } & \text { Treatment effect } \\ \text { SD } & \text { Standard deviation } \\ \text { CI } & 95 \% \text { Confidence interval }\end{array}$

\section{Introduction}

The meniscus plays vital protective roles in the knee, which include supporting joint stability as well as absorption and distribution of loads that are generated during normal weight-bearing and gait [3]. Depending on their size and location, meniscal tears can disrupt these protective functions, resulting in altered joint biomechanics and increased contact pressure on the articular cartilage and bone, with pain and disability as common outcomes [9, 23, 26]. A 2019 systematic review and meta-analysis of asymptomatic, uninjured knees reported that a meniscal tear was observed via magnetic resonance imaging (MRI) in $4 \%$ of individuals under 40 years of age and 19\% of individuals at least 40 years of age [8]. Among patients with knee pain, the incidence of meniscal tears on MRI has been reported to exceed $70 \%$ [10]. Multiple studies have shown that torn or dysfunctional menisci may be associated with impaired mobility, loss of articular cartilage, and the development of radiographic knee osteoarthritis [11, 14, 18, 28]. Thus, maintaining meniscal function is critical to supporting mobility and the long-term health of the knee.

Partial meniscectomy is the most common surgical treatment for symptomatic, irreparable meniscus tears, with 760,000 outpatient procedures performed annually in the US [17]. The principal goals of partial meniscectomy are to relieve knee pain and functional limitations through the surgical removal of loose, unstable meniscal fragments and smoothing of frayed edges to prevent additional tearing.
Evidence from randomized controlled trials (RCT) has suggested that partial meniscectomy may not be superior to non-surgical care alone or sham surgery, but some trials had important study design limitations and high cross-over rates $[1,7,27,32,33,35,36]$. Nonetheless, expert consensus statements and professional societies recommend partial meniscectomy for patients with irreparable meniscus tears and mechanical knee symptoms that are refractory to at least 3 months of non-surgical care [5, 19]. For these patients, partial meniscectomy is safe, with serious complications occurring in less than $1 \%$ of cases [2].

Clinical studies have shown that $6-25 \%$ of patients who previously received a partial meniscectomy experience persistent or recurrent pain within 1-2 years after surgery [20, 33, 37]. Moreover, arthroscopic partial meniscectomy is associated with a significantly increased risk of radiographic osteoarthritis following surgery [24, 29]. Current treatment options for patients with knee pain and impaired mechanical function following partial meniscectomy are limited. In addition, there are no widely accepted clinical practice guidelines that result in relief for these patients. Therefore, there is a critical medical need for effective treatment options that relieve persistent pain, restore meniscal function, and potentially slow the long-term risk of degenerative knee conditions in patients with knee pain following a partial meniscectomy, but who are not clinically ready or indicated for joint replacement.

A synthetic medial meniscus implant (NUsurface ${ }^{\circledR}$, Active Implants LLC, Memphis, TN) was designed to replace previously resected meniscal tissue and provide relief from knee pain and impaired function in patients with knee symptoms following partial meniscectomy. The medial meniscus replacement (MMR) implant was designed to mimic the biomechanical function of the natural medial meniscus, is composed of a pliable yet durable polycarbonate-urethane (PCU) polymer with embedded ultrahigh molecular weight polyethylene (UHMWPE) reinforcement fibers, and does not require fixation to bone or soft tissue [31]. Previous studies have shown that the MMR implant mimics the motion of the native meniscus in the knee joint during flexion, restores pressure distribution and force transmission in the meniscectomized knee to near normal levels, and maintains normal knee kinematics and joint space [31]. This multicenter RCT was designed to test the hypothesis that placement of the MMR implant is a superior treatment strategy compared to non-surgical care for patients with knee pain following one or more previous partial meniscectomies. This study reports the 1-year midpoint results from this 2-year clinical trial. 


\section{Materials and methods}

VENUS (Verifying the Effectiveness of the NUsurface ${ }^{\circledR}$ System, NCT02108496) is a prospective, multicenter, superiority, randomized controlled trial. Prior to patient recruitment, the trial protocol was approved by the Institutional Review Board at each trial site. All subjects gave written informed consent prior to enrollment. The trial was conducted in accordance with the principles of the Declaration of Helsinki and good clinical practice.

Eligible patients were randomized in a 1:1 ratio to receive either the investigational MMR implant or non-surgical care. Randomization was performed using a centralized, web-based, block-randomization system that enabled random treatment assignment from a secure web portal at each study site. Subjects were enrolled at 11 sites in the US from 2014 to 2018 . Trial sites were monitored periodically to confirm that written informed consent was obtained for all participants and to ensure adherence to the trial plan, follow-up schedules, accurate recording of clinical data, and $100 \%$ source verification of the study data. No analyses of the outcome data occurred before subject enrollment were complete.

\section{Participants}

All subjects underwent a complete medical examination and MRI of the index knee before participation. Eligible subjects were between 30 and 75 years of age, had knee pain $(\leq 75$ on a 100-point scale, where 100 is "no pain"), had a medial partial meniscectomy at least 6 months prior to evaluation as confirmed by patient history and MRI, had neutral alignment within $5^{\circ}$ of the mechanical axis, had at least $2 \mathrm{~mm}$ of intact medial meniscal rim, understood the English language, were willing to be randomized into either arm of the trial, and were able to complete trial follow-up visits. Patients were excluded if they had any of the following: evidence of Outerbridge Grade IV cartilage loss on the medial tibial plateau or medial femoral condyle, Outerbridge Grade III or IV cartilage loss in the lateral compartment, detachment of the posterior medial meniscal root, $>5^{\circ}$ of varus or valgus knee deformity, an anterior cruciate ligament reconstruction surgery within the previous 9 months, or body mass index (BMI) exceeding $32.5 \mathrm{~kg} / \mathrm{m}^{2}$. A full list of the inclusion and exclusion criteria are published with the trial registration (ClinicalTrials.gov: NCT02108496).

\section{Treatment groups}

Non-surgical care treatments were prescribed upon randomization. A specific treatment algorithm was not defined in the protocol. Rather, surgeons followed their standard clinical practice for the treatment of post-partial meniscectomy knee pain and tailored the non-surgical care to address the needs and symptoms of each subject. Over time, non-surgical care could be modified to meet changing needs and symptoms. The non-surgical treatment options included intra-articular injections of hyaluronic acid and/or corticosteroids; prescription and/or non-prescription oral medications; physical therapy, low-impact aerobic exercise, and/or strength training; ice and/or heat therapy; unloader knee braces, compression sleeves, crutches and/or canes; weight loss; activity restrictions; and/or shoe inserts or other orthotic devices. The following options were not allowed in the non-surgical care arm: platelet-rich plasma injections, acupuncture, other devices that do not have regulatory approval, and all surgical treatments on index knees.

For the surgical arm, the MMR implant was used. The synthetic MMR implant (NUsurface ${ }^{\circledR}$ Meniscus Implant; Active Implants LLC; Memphis, TN) is made from a durable polycarbonate-urethane polymer (Bionate ${ }^{\circledR} 80 \mathrm{~A}$ PCU; DSM Biomedical; Exton, PA) and contains circumferential reinforcement fibers composed of ultrahigh molecular weight polyethylene (UHMWPE; Dyneema Purity ${ }^{\circledR}$ Fibers; DSM Biomedical; Exton, PA). The MMR implant replaces meniscal tissue in the medial compartment (Fig. 1) and does not require fixation to soft tissue or bone. The surgical procedure involves an arthroscopic sub-total meniscectomy of the medial meniscus, leaving a vertical 2-4 mm meniscal rim, and an arthrotomy of $4-8 \mathrm{~cm}$ to complete the joint preparation and insert the meniscus implant. The MMR implant is available in seven sizes; the target size for each patient is selected preoperatively based on MRI and confirmed intraoperatively using a radiopaque trial implant and fluoroscopy prior to placement of the definitive implant.

Immediate post-operative rehabilitation included compression bandage, knee immobilization for ambulation with weight-bearing as tolerated, and range-of-motion exercises 3-4 times/day. Approximately 1-2 weeks after surgery, patients discontinued knee immobilization with full weight bearing as tolerated, and quadriceps strengthening exercises. By 2-6 weeks after surgery, cycling, straight leg, and closed chain exercises were permitted with full range of motion. After 6 weeks, low-impact activities and open chain exercises were permitted. Subjects in both treatment groups were recommended to not engage in higher impact activities such as contact sports, running/jogging, soccer, or skiing, as a few examples.

\section{Follow-up and outcome measures}

Subjects completed clinical follow-up visits at 1.5 months, 6 months, and 1 year after treatment initiation; subjects will continue follow-up through at least 2 years. The primary 
Fig. 1 Magnetic resonance imaging (MRI) of a knee before (pre-op; a and c) and after (post-op; b and d) implantation of the MMR implant from the coronal (a, b) and sagittal (c, d) views. Arrows indicate the MMR implant

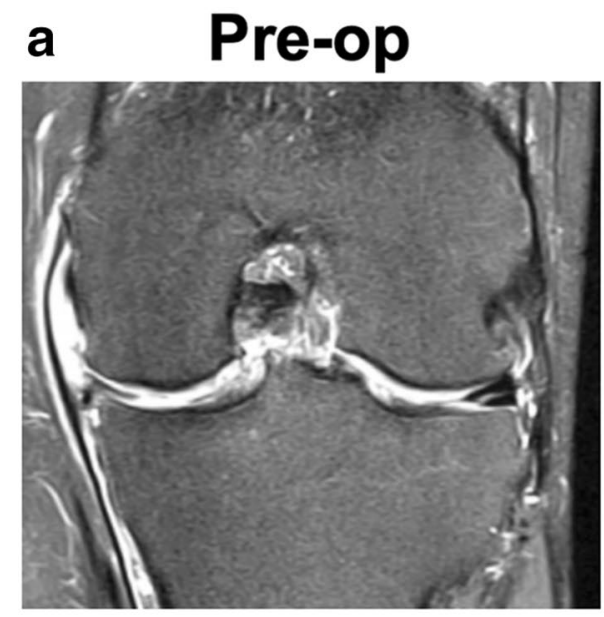

b Post-op
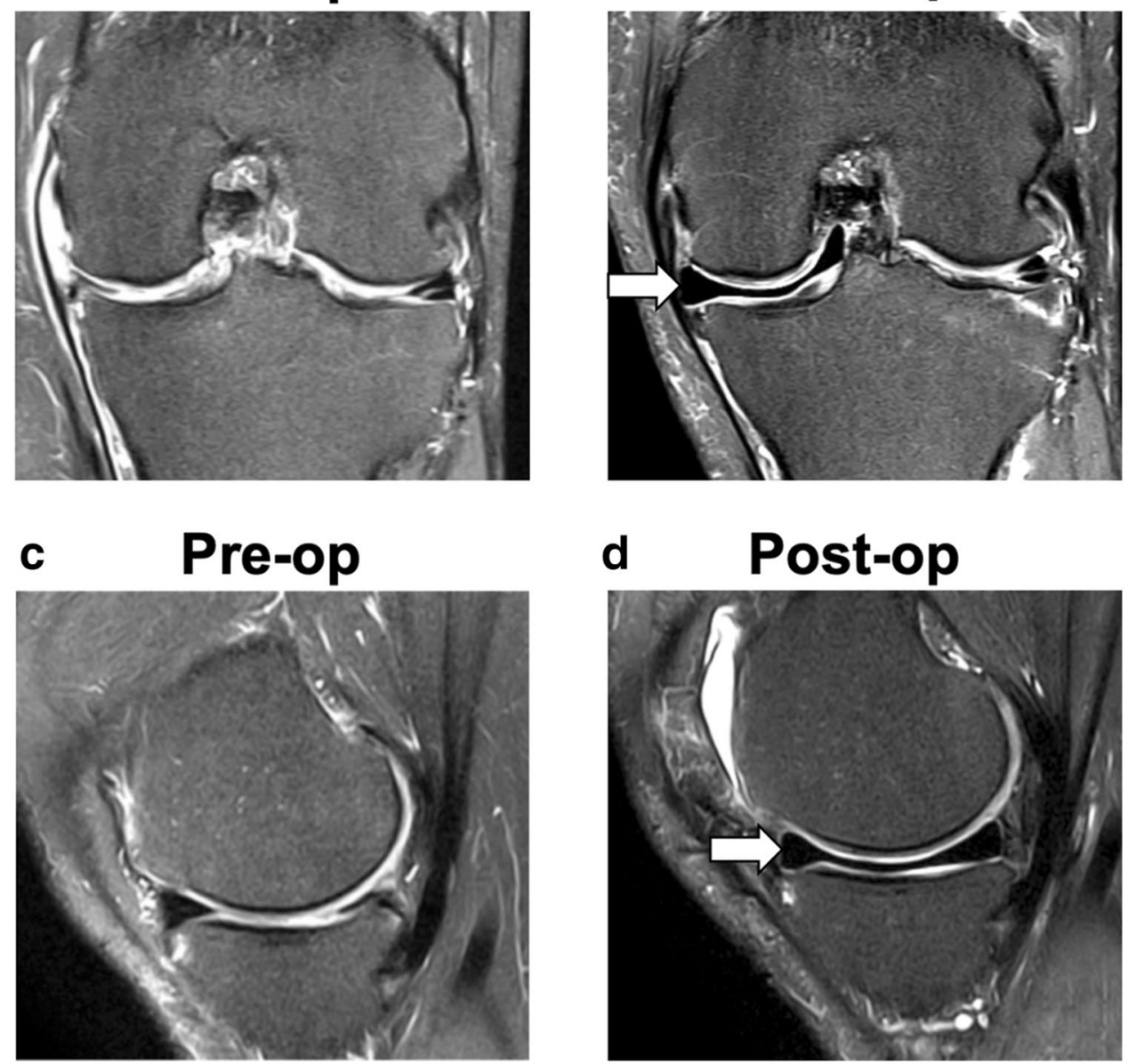

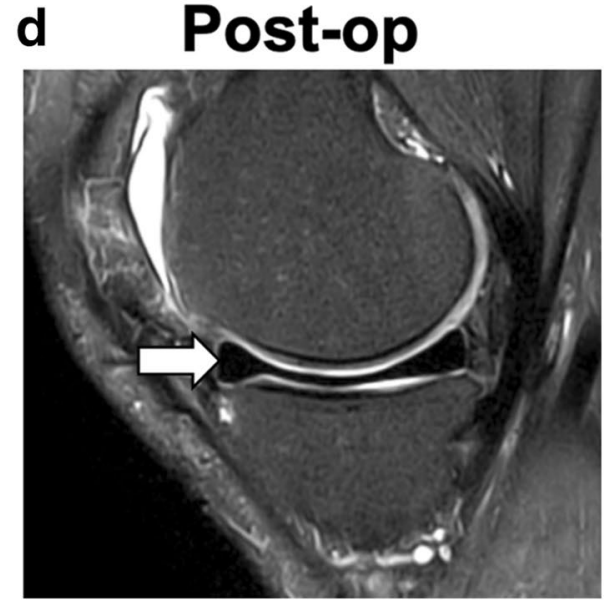

trial endpoint was defined as a composite overall success measure at 2 years of follow-up. Overall success was defined as improvement in the Knee injury and Osteoarthritis Outcome Score (KOOS) subscales of KOOS Pain and KOOS Overall, no MMR implant removal, and an MRI confirming that the MMR implant is in one piece and not subluxed. Overall success will be assessed for superiority at the 2-year primary endpoint. In this 1-year midpoint analysis, improvement in knee-specific pain, function, and quality of life were assessed using the 5 KOOS subscales of pain, symptoms, activities of daily living (ADL), sports and recreation, and quality of life (QOL) as well as the average of the five subscales (KOOS Overall) [30]. KOOS Pain and KOOS Overall were the primary outcome measures, as they will contribute to the primary trial endpoint of overall success. Responders were calculated for each KOOS domain and were defined as subjects who achieved $\mathrm{a} \geq 20$-point improvement from baseline to 1 year of follow-up, or a score at 1 year exceeding thresholds defined for a symptomatic knee [15]. A 10-point improvement in KOOS Pain represents the minimum change that is required to detect a clinically significant improvement from the patient's perspective [22].
Additional validated patient-reported outcome measures included the Visual Analog Scale (VAS) for leg pain, the Western Ontario Meniscal Evaluation Tool (WOMET), and the International Knee Documentation Committee (IKDC) score [13].

Following randomization, subjects were not permitted to cross-over between treatment groups; however, subjects were permitted to cease treatment with non-surgical care or the MMR implant and receive alternative treatments after withdrawing from the clinical trial. Treatment cessation was defined as any subject who decided to discontinue the allocated per-protocol treatment to undergo a surgical procedure on the index knee (non-surgical care group) or undergo permanent removal of the MMR implant (MMR group). Following treatment cessation, patient-reported outcomes were not collected, because they no longer represented the allocated per-protocol treatment (i.e. non-surgical care or retained MMR implant). However, in the MMR group, subjects were permitted to remain in the study and undergo subsequent surgical procedures on the index knee to exchange or reposition the device, as well as other surgeries, as long as the MMR implant was not permanently removed. For MMR 
subjects who underwent subsequent surgical procedures on index knees, patient-reported outcomes were collected after the subsequent surgery and included in the data analysis.

\section{Statistical analysis}

Baseline characteristics were compared between the MMR and non-surgical care groups using two-sample two-sided Student's $t$ tests, two-sided Mann-Whitney test, Fisher's exact test, or Chi-square test. Within-group and betweengroup comparisons of KOOS, VAS, WOMET, and IKDC scores from baseline to each follow-up timepoint were analyzed using a repeated measures mixed-effects model plus Sidak's multiple comparisons test. The repeated measures mixed-effects model accommodates missing values by accounting for within-subject correlation resulting from multiple measures within individual subjects. Sidak's test was used to adjust for the multiple comparisons made between treatment groups across time points. The times from treatment initiation to treatment cessation were compared between groups using a two-sided Student's $t$ test. The rates of KOOS responders and treatment cessation were compared between groups using Fisher's exact test. The D'Agostino and Pearson normality test was used to determine if data satisfied assumptions of normality. A two-sided $P$ value of $\leq 0.05$ was considered statistically significant. The required sample size for this trial was calculated to be 52 subjects per group based on assumptions of $80 \%$ power and a type I error rate of $5 \%$ to detect a statistically significant difference in a binary outcome of overall treatment success using a Chi-Square test of two proportions. Assuming a loss to follow-up of up to $15 \%$, enrollment targets were at least 62 subjects per group.

\section{Results}

One hundred twenty-seven subjects were randomized and received either surgical (MMR) treatment $(n=61)$ or nonsurgical care ( $n=66$; Fig. 2 ). The baseline patient characteristics were similar between the MMR and non-surgical care groups (Table 1). Subjects were an average of 50 years old, mostly (76\%) men, and had an average BMI of $26.8 \mathrm{~kg} /$ $\mathrm{m}^{2}$. Seventy percent of subjects had one previous partial meniscectomy and $30 \%$ had two or more previous partial meniscectomies.

Non-surgical treatment was designed to mimic the surgeon's current standard non-operative treatment for persistent post-partial meniscectomy knee pain, was individually tailored to each subject, and varied from surgeon to surgeon (Table 2). The average number of hyaluronic acid injections and corticosteroid injections among subjects who received them was 3.1 (median: 3; range: 1-6) and 1.3 (median: 1; range: $1-3)$, respectively.

\section{KOOS}

Both surgical and control groups showed improvement in KOOS scores at 1 year compared with baseline $(P<0.05)$. The magnitude of improvement from baseline to 1 year of follow-up was significantly greater in the MMR group compared to non-surgical care for the primary outcomes of KOOS Overall (Fig. 3a; $P=0.027$ ) and KOOS Pain (Fig. 3b; $P=0.013$ ). Improvements were also significantly greater in the MMR group for KOOS ADL and QOL, but the differences were not statistically significant for KOOS Symptoms or Sports and Recreation (Fig. 3c-f). The KOOS responder rate was significantly higher in the MMR group for KOOS Overall, ADL, Sports and Recreation, and QOL (Fig. 4).

\section{VAS pain, WOMET, and IKDC}

VAS pain and WOMET scores were significantly better among MMR subjects compared to the non-surgical care group at 6 months and 1 year. Specifically, the magnitude of improvement from baseline to 1 year was significantly greater in the MMR group vs non-surgical care for VAS Pain $(P=0.004)$, WOMET $(P=0.002)$ and IKDC scores $(P=0.03)$ compared to non-surgical care (Additional File 1).

\section{Treatment cessation}

There was a higher frequency of treatment cessation in the non-surgical care group $(8 / 55 ; 14.5 \%)$ compared to the MMR group $(3 / 61 ; 4.9 \%)$, but the difference was not statistically significant. Among control subjects, treatment cessation was associated with a variety of arthroscopic procedures, high tibial osteotomy, or unicondylar knee replacement; the three MMR subjects ceased treatment following device rotation or subluxation (Table 3 ).

\section{Subsequent surgical procedures}

In addition to the treatment cessations described above, there were eight subsequent surgical procedures in seven MMR subjects $(11.5 \%)$ over 1 year of follow-up (Table 4). One subject had two subsequent surgical procedures during the 1st year to reposition the implant after it rotated as a result of traumatic twisting of the knee. Following this second incident, the implant was exchanged with a larger size to avoid instability. In addition, two more subjects underwent a device exchange procedure due to implant damage and dislocation during exercise or sports.

Four MMR subjects underwent other subsequent surgical procedures that did not involve repositioning or exchange of 


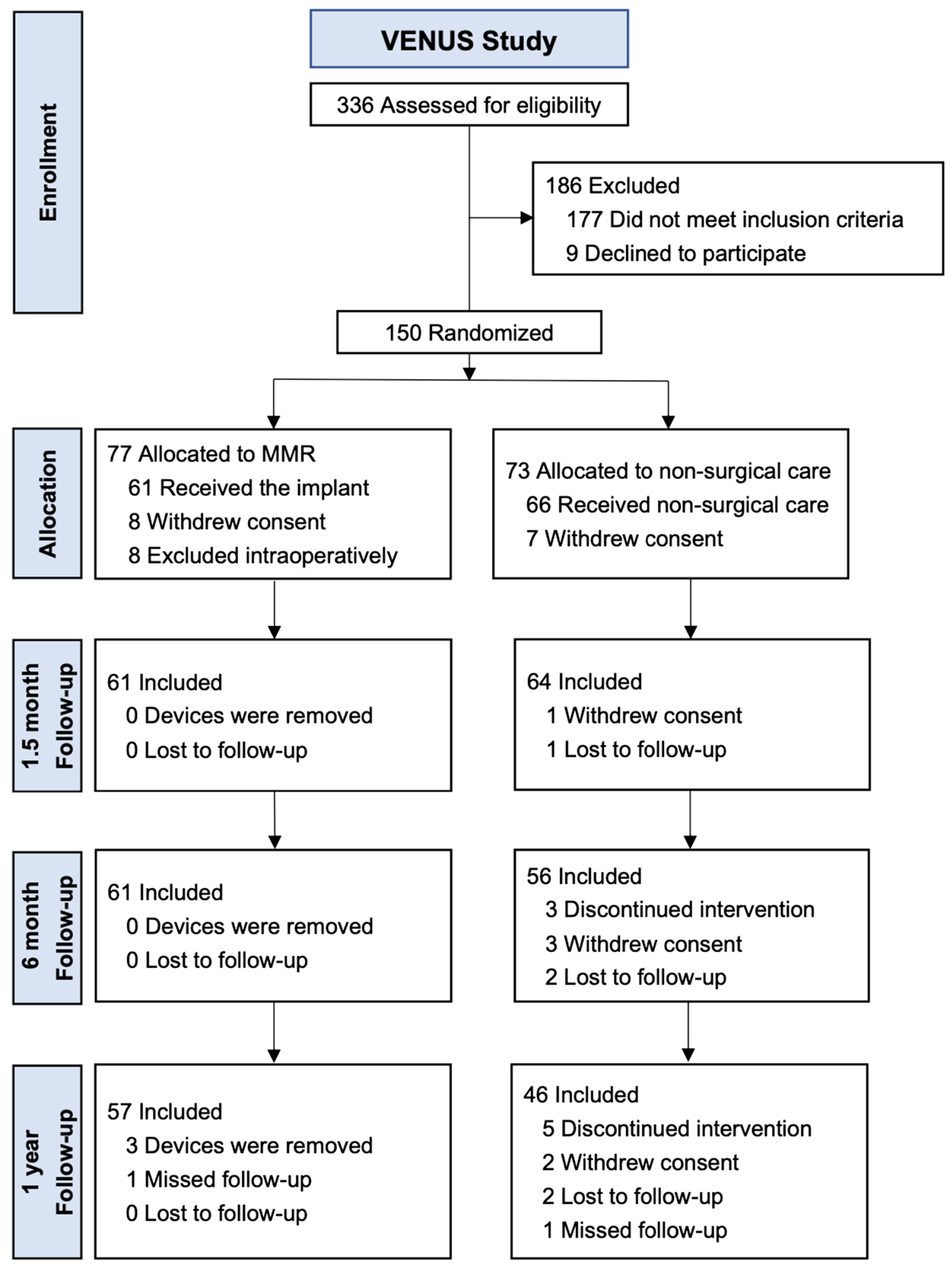

Fig. 2 CONSORT flow diagram of patient allocation and follow-up

the MMR implant. The implant was confirmed to be intact and in a stable position in these cases. One subject underwent a synovectomy, chondroplasty, and notchplasty with removal of osteophytes for recurrent effusion. Three subjects underwent lysis of adhesion or notchplasty to address patient-reported difficultly with full knee flexion. 
Table 1 Patient baseline characteristics

\begin{tabular}{llll}
\hline Characteristic & $\begin{array}{l}\text { MMR } \\
(n=61)\end{array}$ & $\begin{array}{l}\text { Non-surgical } \\
(n=66)\end{array}$ & $P$ value \\
\hline Age & $51.0 \pm 11.2$ & $49.8 \pm 10.3$ & n.s \\
Body mass index (BMI) & $26.8 \pm 3.2$ & $26.8 \pm 3.6$ & n.s \\
Male Gender- $n(\%)$ & $48(78.7 \%)$ & $48(72.7 \%)$ & n.s \\
Left index knee— $n(\%)$ & $31(50.8 \%)$ & $31(47.0 \%)$ & n.s \\
Number of previous partial meniscectomies & & & n.s \\
One— $n(\%)$ & $42(68.9 \%)$ & $46(69.7 \%)$ & \\
Two-n(\%) & $11(18.0 \%)$ & $14(21.2 \%)$ & \\
Three or more- $n(\%)$ & $8(13.1 \%)$ & $6(9.1 \%)$ & \\
Median (range) months since last meniscectomy & $34(7-313)$ & $35(5-425)$ & n.s \\
KOOS & & & \\
Pain & $52.1 \pm 11.2$ & $54.2 \pm 15.6$ & n.s \\
Symptoms & $59.4 \pm 15.3$ & $62.6 \pm 16.5$ & n.s \\
ADL & $63.4 \pm 16.0$ & $65.3 \pm 19.9$ & n.s \\
Sports and recreation & $35.5 \pm 22.2$ & $39.3 \pm 21.7$ & n.s \\
QOL & $27.6 \pm 16.9$ & $30.0 \pm 13.5$ & n.s \\
Overall & $47.6 \pm 12.6$ & $50.3 \pm 14.3$ & n.s \\
VAS pain & $53.3 \pm 20.5$ & $51.0 \pm 23.7$ & n.s \\
WOMET & $36.3 \pm 16.2$ & $39.1 \pm 16.6$ & n.s \\
IKDC & $42.4 \pm 13.2$ & $45.4 \pm 13.9$ & n.s \\
\hline
\end{tabular}

Values are mean \pm standard deviation or number $(n)$ and percent $(\%)$ unless otherwise specified n.s. not significant

\begin{tabular}{ll}
\hline & Intervention no. (\%) \\
\hline Intra-articular hyaluronic acid injection & $32(48 \%)$ \\
Physical therapy, non-weight-bearing or weight-bearing exercises & $30(45 \%)$ \\
Compression sleeves or unloader braces & $29(44 \%)$ \\
Prescription or non-prescription NSAIDs & $23(35 \%)$ \\
Activity limitation & $22(33 \%)$ \\
Ice or heat therapy & $21(32 \%)$ \\
Intra-articular corticosteroid injection & $8(12 \%)$ \\
Body weight reduction & $5(8 \%)$ \\
Non-prescription drugs, creams, vitamins, or supplements & $4(6 \%)$ \\
Shoe orthotic devices & $3(5 \%)$ \\
\hline
\end{tabular}

Values do not sum to $100 \%$ because many subjects were prescribed multiple types of non-surgical care treatments

\section{Discussion}

The most important finding of the present study was the MMR implant resulted in significantly greater improvements in the primary outcome measures of KOOS Pain and KOOS Overall at 1 year of follow-up compared to standard non-surgical care in patients with persistent knee pain following previous partial meniscectomy. Furthermore, a greater proportion of subjects in the non-surgical care group discontinued treatment and underwent a subsequent surgical procedure compared to the proportion of subjects in the MMR group who had the device permanently removed; however, the difference was not statistically significant. These findings are of important clinical relevance considering the highly limited treatment options for this patient population. These results may help to inform patients and healthcare providers regarding treatment decisions and to set expectations for the 1st year of treatment.

Currently, there are limited treatment options for patients with knee pain following partial meniscectomy. 

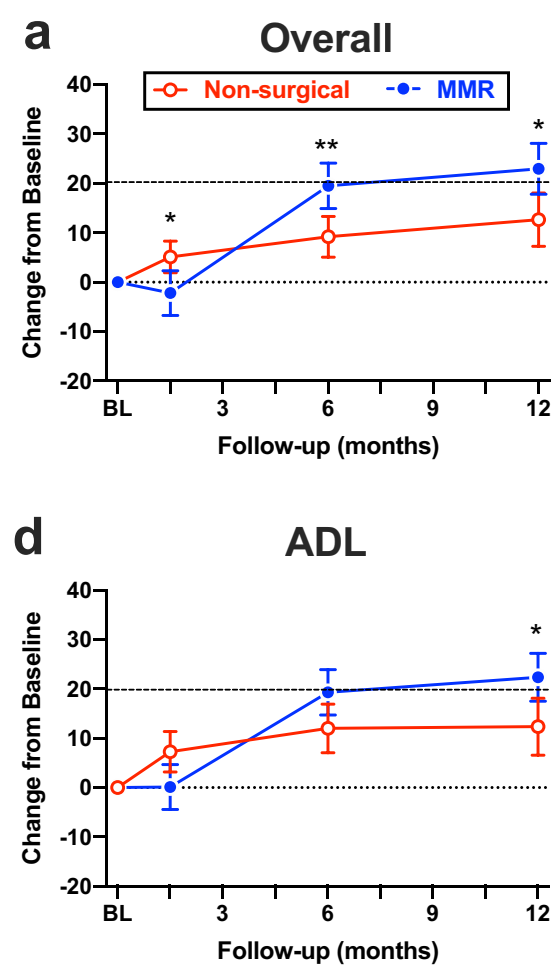

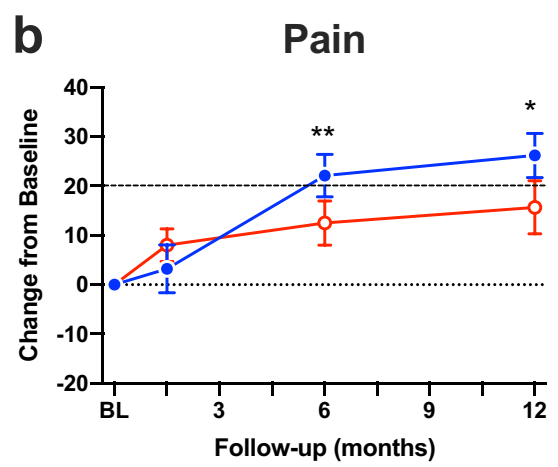

C
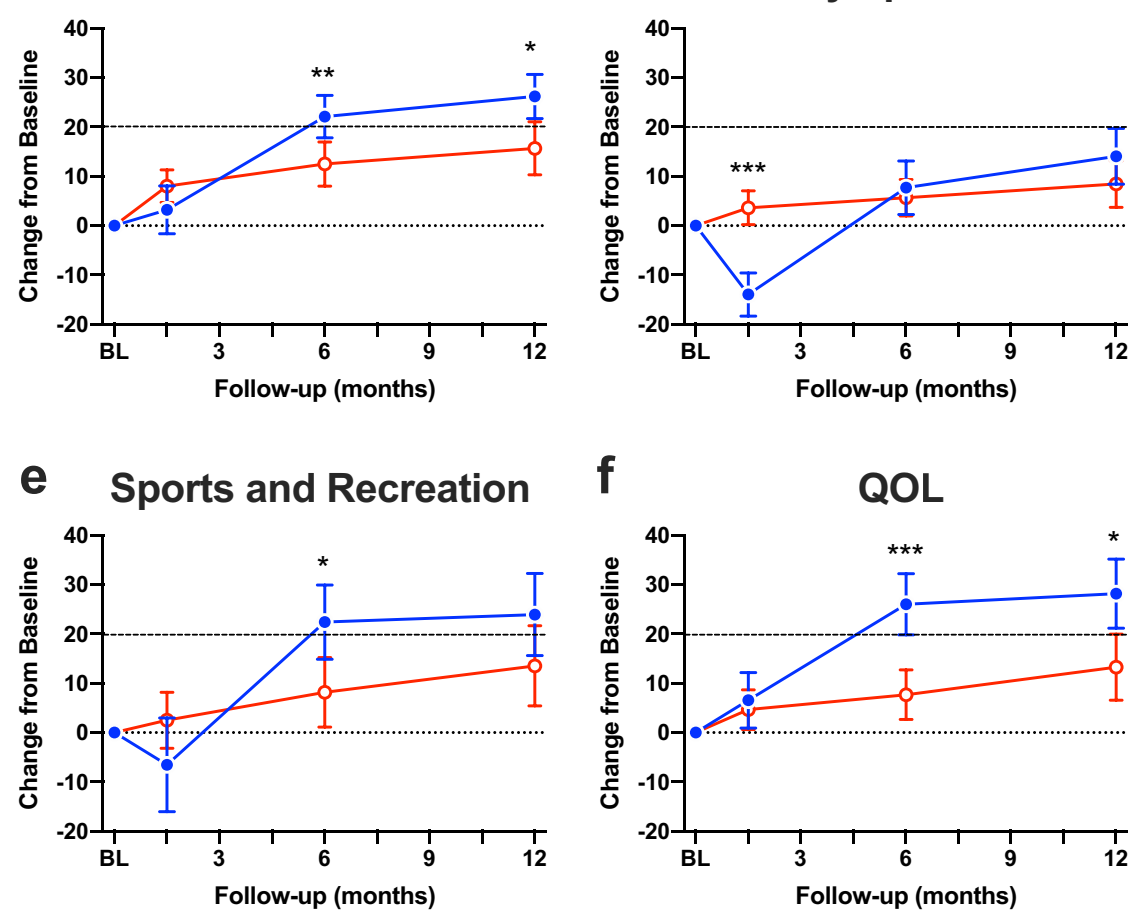

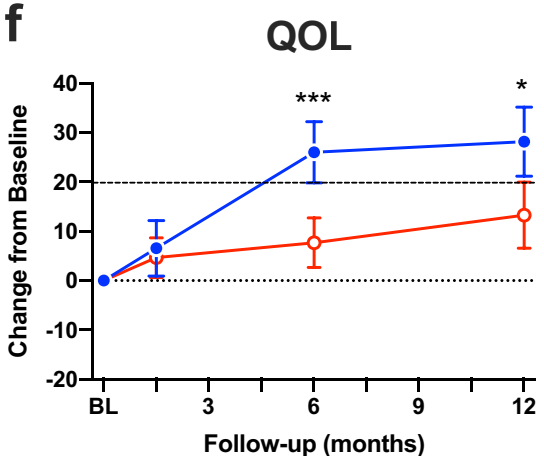

Fig. 3 Change in KOOS subscales. Magnitude of change from baseline (BL) to 1 year of follow-up in KOOS Overall (a), pain (b), symptoms (c), activities of daily living (ADL; d), sports and recreation (e), and quality of life (QOL; $\mathbf{f})$. Symbols indicate the mean and error bars are $95 \%$ confidence intervals. Open red symbols represent

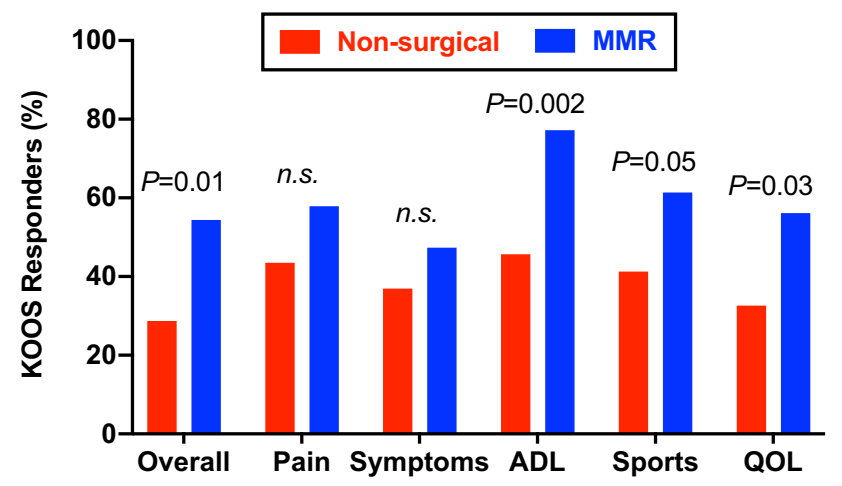

Fig. 4 Percentage of responders in each KOOS subscale. The percentage of subjects who achieved $\mathrm{a} \geq 20$-point improvement in each KOOS subscale at 1 year, or a 1 -year score exceeding thresholds defined for a symptomatic knee. Red bars represent the non-surgical care group and blue bars represent the medial meniscus replacement (MMR) group. n.s. not significant

Intra-articular corticosteroid injections not only may provide temporary ( $<6$ months) relief from pain, but also can exacerbate the loss of articular cartilage [25]. Hyaluronic acid injections provide transient relief from pain, but require frequent recurrent injections and may not provide a clinically the non-surgical care group and closed blue symbols represent the medial meniscus replacement (MMR) implant group. Higher values indicate better improvement in pain, symptoms, function, or quality of life. *Indicates $P<0.05$, ** indicates $P<0.01$, and $* * *$ indicates $P<0.001$ for MMR group vs non-surgical care group

meaningful benefit immediately after partial meniscectomy $[6,12]$. Meniscal allograft transplantation is associated with significant improvements in knee pain and function in younger patients (mean age: 28 years; range 17-46 years) with symptomatic post-meniscectomy knees [34]. However, allograft transplantation is limited to younger patients ( $<50$ years), by the supply of tissue bank allografts, and can be challenging to match in size and shape to ensure proper fit $[16,21]$. Finally, treatments such as unicompartmental or total knee replacement are intended for patients over 60 years old and those with end-stage degenerative knee conditions, because the risk of revision surgery is approximately $20-35 \%$ for patients aged $50-54$ years [4]. In this study, the MMR implant appears to provide encouraging outcomes at the 1-year midpoint of a 2-year superiority trial, which may indicate that this will be an important treatment option to bridge the current treatment gap for patients with persistent knee pain after partial medial meniscectomy.

There are three notable advantages of the MMR implant. First, the implant does not require fixation or anchoring to bone or soft tissue, which allows the implant to translate through the full range of motion during knee articulation. Of course, the lack of fixation may allow for rotation or subluxation of the implant, and also allows the implant to be readily 
Table 3 Surgical procedures associated with treatment cessation

\begin{tabular}{ll}
\hline Type of surgery & $\begin{array}{l}\text { Unique subjects } \\
n(\%)\end{array}$ \\
\hline MMR group & $3(4.9 \%)$ \\
Permanent implant removal following device rotation & $2(3.3 \%)$ \\
Permanent implant removal following device dislocation & $1(1.6 \%)$ \\
Non-surgical care group & $8(14.5 \%)$ \\
Exploratory arthroscopy & $1(1.8 \%)$ \\
Medial and lateral partial meniscectomy & $1(1.8 \%)$ \\
Medial arthroscopic partial meniscectomy, synovectomy, and chondroplasty & $1(1.8 \%)$ \\
Medial partial meniscectomy and posterolateral corner reconstruction & $1(1.8 \%)$ \\
High tibial osteotomy & $1(1.8 \%)$ \\
Trochlear osteochondral allograft transplantation & $1(1.8 \%)$ \\
Meniscus transplantation & $1(1.8 \%)$ \\
Unicondylar knee replacement & $1(1.8 \%)$ \\
\hline
\end{tabular}

Table 4 Summary of subsequent surgical procedures on index knees in the MMR group

\begin{tabular}{|c|c|c|}
\hline Type of subsequent surgery & $\begin{array}{l}\text { Events } \\
n\end{array}$ & $\begin{array}{l}\text { Unique subjects } \\
n(\%)\end{array}$ \\
\hline Permanent implant removal & 3 & $3(4.9 \%)$ \\
\hline Rotation & 2 & $2(3.3 \%)$ \\
\hline Dislocation & 1 & $1(1.6 \%)$ \\
\hline Implant exchange & 3 & $3(4.9 \%)$ \\
\hline Damage + Dislocation & 2 & $2(3.3 \%)$ \\
\hline Rotation & 1 & $1(1.6 \%)$ \\
\hline Implant repositioned & 1 & $1(1.6 \%)$ \\
\hline Rotation & 1 & $1(1.6 \%)$ \\
\hline Other $^{\mathrm{a}}$ & 4 & $4(6.6 \%)$ \\
\hline
\end{tabular}

${ }^{a}$ Other subsequent surgical procedures included synovectomy, chondroplasty, notchplasty, debridement, or adhesion lysis

exchanged or repositioned during a subsequent surgery if required. Second, the pliable, PCU-based MMR implant mimics the biomechanical function of the native meniscus, conforms to joint surfaces to distribute loads, and has been shown to slow the onset of articular cartilage degeneration in an animal model of meniscectomy [31,38]. With similar biomechanical properties to the native meniscus, mechanical wear and failure of the implant could occur under excessive or abnormal use. Finally, the surgical procedure does not require extensive resection of bone or alteration of the anatomy, allowing for future conversion to a reconstructive surgical procedure, such as a joint replacement, when determined clinically necessary. Therefore, the MMR implant can serve as a bridging treatment by delaying the time between partial meniscectomy and more invasive procedures such as knee replacement.

This trial has several important strengths, including enrollment of patients across 11 trial sites in the United States, strict patient eligibility criteria, and randomized treatment allocation. However, one limitation of the trial is that $16.7 \%$ of subjects in the non-surgical care group were lost to follow-up or withdrew within the 1 st year. In addition, subject blinding was not possible due to the nature of the treatments, which may have exposed the results to potential expectation bias during the collection of patientreported outcomes. Finally, functional assessments focused on patient-reported outcomes and did not include objective measurements, such as range of motion, because patient perception information is an important indicator for determining the clinical relevance and impact of a new therapy.

\section{Conclusions}

Subjects with knee pain following partial meniscectomy who were randomly assigned to receive MMR had significantly greater improvements from baseline in knee pain, activities of daily living, and quality of life at 1 year of follow-up of an ongoing 2-year clinical trial compared to non-surgical care alone. Differences in mechanical symptoms and sports and recreation were not statistically significant between the groups at 1 year.

Supplementary Information The online version contains supplementary material available at https://doi.org/10.1007/s00167-021-06573-0.

Funding Active Implants LLC provided support for the conduct of the study, data analysis, and preparation of this manuscript.

\section{Declarations}

Conflict of interest $\mathrm{KZ}$ reports personal fees from Zimmer Biomet, Aesculap, Cartiheal, and Orthospace outside of the submitted work. $\mathrm{JF}$ reports research support from Episurf, Fidia Pharma, Moximed Inc, Novartis Inc, Samumed Inc, Vericel Inc, Zimmer Biomet, Organogen- 
esis outside of this submitted work. Personal consulting fees, stocks, and/or royalties from Moximed Inc, Vericel Inc, Arthrex, Depuy Syntheses Products Inc, Organogenesis, Biopoly LLC, Medshape Inc, OrthoRegnerative Technologies, Aesculap, Cartiheal, Cook Biotech Inc, JRF Ortho, ZKR Orthopedics Inc, and Regentis Biomaterials outside of the submitted work. Editorial Board for American Journal of Sports Medicine, Cartilage, and Journal of Knee Surgery outside of the submitted work. AG reports personal consulting fees from Moximed, Organogenesis, JRF, Smith and Nephew, and Vericel outside of the submitted work. MD reports salaried employee of Telos Partners, LLC, which received funding from Active Implants to support data analysis and technical writing related to the submitted work. EH reports personal consulting fees from Active Implants outside of the submitted work. CL reports personal fees from Joint Restoration Foundation, Sanumed, and Vericel outside of the submitted work. BM reports minority ownership in Boston Outpatient Surgical Suites and shareholder in Parcus Medical, LLC outside of the submitted work. The other authors report no potential conflicts of interest.

Ethical approval All procedures involving human subjects performed in this study were approved by the Institutional Review Board at each site and were conducted in accordance with the principles of the Declaration of Helsinki and good clinical practice.

Informed consent All subjects gave written informed consent before participation.

Open Access This article is licensed under a Creative Commons Attribution 4.0 International License, which permits use, sharing, adaptation, distribution and reproduction in any medium or format, as long as you give appropriate credit to the original author(s) and the source, provide a link to the Creative Commons licence, and indicate if changes were made. The images or other third party material in this article are included in the article's Creative Commons licence, unless indicated otherwise in a credit line to the material. If material is not included in the article's Creative Commons licence and your intended use is not permitted by statutory regulation or exceeds the permitted use, you will need to obtain permission directly from the copyright holder. To view a copy of this licence, visit http://creativecommons.org/licenses/by/4.0/.

\section{References}

1. Abram SGF, Hopewell S, Monk AP, Bayliss LE, Beard DJ, Price AJ (2020) Arthroscopic partial meniscectomy for meniscal tears of the knee: a systematic review and meta-analysis. Br J Sports Med 54:652-663

2. Abram SGF, Judge A, Beard DJ, Price AJ (2018) Adverse outcomes after arthroscopic partial meniscectomy: a study of 700000 procedures in the national Hospital Episode Statistics database for England. Lancet 392:2194-2202

3. Baron SL, Jazrawi LM (2020) Anatomy and Function. In: Strauss EJ, Jazrawi LM (eds) The management of meniscal pathology: from meniscectomy to repair and transplantation. Springer International Publishing, Cham, pp 1-13

4. Bayliss LE, Culliford D, Monk AP, Glyn-Jones S, Prieto-Alhambra D, Judge A et al (2017) The effect of patient age at intervention on risk of implant revision after total replacement of the hip or knee: a population-based cohort study. Lancet 389:1424-1430

5. Beaufils P, Becker R, Kopf S, Englund M, Verdonk R, Ollivier $M$ et al (2017) Surgical management of degenerative meniscus lesions: the 2016 ESSKA Meniscus Consensus. Joints 5:59-69
6. Bedard NA, Dowdle SB, Anthony CA, DeMik DE, McHugh MA, Bozic KJ et al (2017) The AAHKS Clinical Research Award: what are the costs of knee osteoarthritis in the year prior to total knee arthroplasty? J Arthroplasty 32:S8-S10.e11

7. Berg B, Roos EM, Englund M, Kise NJ, Tiulpin A, Saarakkala S et al (2020) Development of osteoarthritis in patients with degenerative meniscal tears treated with exercise therapy or surgery: a randomized controlled trial. Osteoarthr Cartil 28:897-906

8. Culvenor AG, Oiestad BE, Hart HF, Stefanik JJ, Guermazi A, Crossley KM (2019) Prevalence of knee osteoarthritis features on magnetic resonance imaging in asymptomatic uninjured adults: a systematic review and meta-analysis. Br J Sports Med 53:1268-1278

9. Daney BT, Aman ZS, Krob JJ, Storaci HW, Brady AW, Nakama $G$ et al (2019) Utilization of transtibial centralization suture best minimizes extrusion and restores tibiofemoral contact mechanics for anatomic medial meniscal root repairs in a cadaveric model. Am J Sports Med 47:1591-1600

10. Deshpande BR, Losina E, Smith SR, Martin SD, Wright RJ, Katz JN (2016) Association of MRI findings and expert diagnosis of symptomatic meniscal tear among middle-aged and older adults with knee pain. BMC Musculoskelet Disord 17:154

11. Driban JB, Harkey MS, Barbe MF, Ward RJ, MacKay JW, Davis JE et al (2020) Risk factors and the natural history of accelerated knee osteoarthritis: a narrative review. BMC Musculoskelet Disord 21:332

12. Drobnic M, Ercin E, Gamelas J, Papacostas ET, Slynarski K, Zdanowicz U et al (2019) Treatment options for the symptomatic post-meniscectomy knee. Knee Surg Sports Traumatol Arthrosc 27:1817-1824

13. Dwyer T, Zochowski T, Ogilvie-Harris D, Theodoropoulos J, Whelan D, Chahal J (2020) Determining the patient acceptable symptomatic state for patients undergoing arthroscopic partial meniscectomy in the knee. Am J Sports Med 48:847-852

14. Englund M, Guermazi A, Roemer FW, Aliabadi P, Yang M, Lewis CE et al (2009) Meniscal tear in knees without surgery and the development of radiographic osteoarthritis among middle-aged and elderly persons: the multicenter osteoarthritis study. Arthritis Rheum 60:831-839

15. Englund M, Roos EM, Lohmander LS (2003) Impact of type of meniscal tear on radiographic and symptomatic knee osteoarthritis: a sixteen-year followup of meniscectomy with matched controls. Arthritis Rheum 48:2178-2187

16. Gitelis ME, Frank RM, Meyer MA, Cvetanovich G, Cole BJ (2018) 5 Points on meniscal allograft transplantation. Am J Orthop (Belle Mead NJ). https://doi.org/10.12788/ajo.2018. 0069

17. Hall MJ, Schwartzman A, Zhang J, Liu X (2017) Ambulatory surgery data from hospitals and ambulatory surgery centers: United States, 2010. Natl Health Stat Report 1-15

18. Hare KB, Stefan Lohmander L, Kise NJ, Risberg MA, Roos EM (2017) Middle-aged patients with an MRI-verified medial meniscal tear report symptoms commonly associated with knee osteoarthritis. Acta Orthop 88:664-669

19. Hohmann E, Angelo R, Arciero R, Bach BR, Cole B, Cote M et al (2020) Degenerative meniscus lesions: an expert consensus statement using the modified Delphi technique. Arthroscopy 36:501-512

20. Katz JN, Brophy RH, Chaisson CE, de Chaves L, Cole BJ, Dahm DL et al (2013) Surgery versus physical therapy for a meniscal tear and osteoarthritis. N Engl J Med 368:1675-1684

21. Lee DH (2018) Incidence and extent of graft extrusion following meniscus allograft transplantation. Biomed Res Int 2018:5251910

22. Liu JN, Gowd AK, Redondo ML, Christian DR, Cabarcas BC, Yanke AB et al (2019) Establishing clinically significant outcomes 
after meniscal allograft transplantation. Orthop J Sports Med 7:2325967118818462

23. Logan CA, Aman ZS, Kemler BR, Storaci HW, Dornan GJ, LaPrade RF (2019) Influence of medial meniscus bucket-handle repair in setting of anterior cruciate ligament reconstruction on tibiofemoral contact mechanics: a biomechanical study. Arthroscopy 35:2412-2420

24. Longo UG, Ciuffreda M, Candela V, Rizzello G, D’Andrea V, Mannering $\mathrm{N}$ et al (2019) Knee osteoarthritis after arthroscopic partial meniscectomy: prevalence and progression of radiographic changes after 5 to 12 years compared with contralateral knee. J Knee Surg 32:407-413

25. McAlindon TE, LaValley MP, Harvey WF, Price LL, Driban JB, Zhang M et al (2017) Effect of intra-articular triamcinolone vs saline on knee cartilage volume and pain in patients with knee osteoarthritis: a randomized clinical trial. JAMA 317:1967-1975

26. Nakama GY, Kaleka CC, Franciozi CE, Astur DC, Debieux P, Krob JJ et al (2019) Biomechanical Comparison of vertical mattress and cross-stitch suture techniques and single- and doublerow configurations for the treatment of bucket-handle medial meniscal tears. Am J Sports Med 47:1194-1202

27. Noorduyn JCA, Glastra van Loon T, van de Graaf VA, Willigenburg NW, Butter IK, Scholten-Peeters GGM et al (2020) Functional outcomes of arthroscopic partial meniscectomy versus physical therapy for degenerative meniscal tears using a patientspecific score: a randomized controlled trial. Orthop J Sports Med 8:2325967120954392

28. Pihl K, Englund M, Lohmander LS, Jorgensen U, Nissen N, Schjerning J et al (2017) Signs of knee osteoarthritis common in 620 patients undergoing arthroscopic surgery for meniscal tear. Acta Orthop 88:90-95

29. Roemer FW, Kwoh CK, Hannon MJ, Hunter DJ, Eckstein F, Grago $\mathrm{J}$ et al (2017) Partial meniscectomy is associated with increased risk of incident radiographic osteoarthritis and worsening cartilage damage in the following year. Eur Radiol 27:404-413

30. Roos EM, Hare KB, Nielsen SM, Christensen R, Lohmander LS (2018) Better outcome from arthroscopic partial meniscectomy than skin incisions only? A sham-controlled randomised trial in patients aged 35-55 years with knee pain and an MRI-verified meniscal tear. BMJ Open 8:e019461

31. Shemesh M, Shefy-Peleg A, Levy A, Shabshin N, Condello V, Arbel R et al (2020) Effects of a novel medial meniscus implant on the knee compartments: imaging and biomechanical aspects. Biomech Model Mechanobiol. https://doi.org/10.1007/ s10237-020-01323-6

32. Sihvonen R, Paavola M, Malmivaara A, Itala A, Joukainen A, Kalske J et al (2020) Arthroscopic partial meniscectomy for a degenerative meniscus tear: a 5 year follow-up of the placebosurgery controlled FIDELITY (Finnish Degenerative Meniscus Lesion Study) trial. Br J Sports Med 54:1332-1339

33. Sihvonen R, Paavola M, Malmivaara A, Itala A, Joukainen A, Nurmi H et al (2018) Arthroscopic partial meniscectomy versus placebo surgery for a degenerative meniscus tear: a 2-year followup of the randomised controlled trial. Ann Rheum Dis 77:188-195

34. Smith NA, Parsons N, Wright D, Hutchinson C, Metcalfe A, Thompson $\mathrm{P}$ et al (2018) A pilot randomized trial of meniscal allograft transplantation versus personalized physiotherapy for patients with a symptomatic meniscal deficient knee compartment. Bone Joint J 100-B:56-63

35. Smoak JB, Matthews JR, Vinod AV, Kluczynski MA, Bisson LJ (2020) An up-to-date review of the meniscus literature: a systematic summary of systematic reviews and meta-analyses. Orthop J Sports Med 8:2325967120950306

36. van de Graaf VA, Noorduyn JCA, Willigenburg NW, Butter IK, de Gast A, Mol BW et al (2018) Effect of early surgery vs physical therapy on knee function among patients with nonobstructive meniscal tears: the ESCAPE randomized clinical trial. JAMA 320:1328-1337

37. Yim JH, Seon JK, Song EK, Choi JI, Kim MC, Lee KB et al (2013) A comparative study of meniscectomy and nonoperative treatment for degenerative horizontal tears of the medial meniscus. Am J Sports Med 41:1565-1570

38. Zur G, Linder-Ganz E, Elsner JJ, Shani J, Brenner O, Agar G et al (2011) Chondroprotective effects of a polycarbonate-urethane meniscal implant: histopathological results in a sheep model. Knee Surg Sports Traumatol Arthrosc 19:255-263

Publisher's Note Springer Nature remains neutral with regard to jurisdictional claims in published maps and institutional affiliations. 